\title{
¿ES POSIBLE LA AGRICULTURA ORGÁNICA EN MARINILLA?: ENTRE LA CAPACIDAD DE LOS RECURSOS Y LA VOLUNTAD POLÍTICA, SE HACE CAMINO*
}

\author{
Luz Dolly Lopera García* \\ Dora Cristina Salgado Cañaveral ${ }^{\star \star *}$ \\ Raúl Velásquez Vélez $z^{\star \star \star *}$
}

Recibido: agosto 28 de 2011

Aceptado: octubre 18 de 2011

\section{RESUMEN}

El objetivo del artículo es describir algunas características socioeconómicas y técnico-agrícolas de 28 unidades de producción de frutas y verduras agrupadas en asociaciones del municipio de Marinilla, Antioquia, Colombia. Se utilizó una metodología en la que predomina el enfoque cuantitativo, complementada con búsqueda bibliográfica documental y entrevistas a funcionarios de la Unidad Municipal de Asistencia Técnica Agropecuaria y Secretaría de Agricultura. El resultado más relevante sugiere que en su mayoría las unidades generan productos para la subsistencia de los agricultores y sus familias y para la venta, en pequeño volumen, hacia el mercado local. También se evidenció que en su mayoría las unidades no desarrollan producción orgánica y en caso de que se les exija algún requisito de calidad, para la venta de sus productos en el mercado interno, no se da la importancia debida a este tipo de producción.

\section{PALABRAS CLAVE}

Asociaciones, agricultura, agricultura y comercio internacional, política alimentaria.

\section{CLASIFICACIÓN JEL} D71, Q17, Q18, Q19

\section{CONTENIDO}

Introducción; 1. La producción orgánica como sustrato del desarrollo humano y sustentable; 2. La certificación de la producción orgánica para un mercado exigente; 3. Condiciones de la agricultura orgánica en Marinilla: posibilidades para la certificación; 4. Conclusiones; Bibliografía.

\footnotetext{
El artículo es el resultado de la investigación "Características económicas, organizacionales, ambientales y técnico-agrícola de las pequeñas y medianas empresas de productores de frutas y verduras del municipio de Marinilla, 2008", financiada por la Fundación Universitaria Luis Amigó (FUNLAM) en el 2008, realizada por los docentes Luz Dolly Lopera García del grupo de investigación de Economía Solidaria (ECOSOL) clasificado en Colciencias, categoría B y en calidad de coinvestigadores, Dora Cristina Salgado Cañaveral, Raúl Velásquez Vélez, Gloria Echeverri Lopera de la Facultad de Ciencias Administrativas, Económicas y Contables, FUNLAM, Medellín, Colombia; y Johan Ramirez Canedo de la Corporación de Altos Estudios Equinos de Colombia, Caequinos, Medellín, Colombia.

* Economista, especialista en Administración de Servicios de Salud y magíster en Salud Pública, Universidad de Antioquia, Medellín, Colombia. Miembro del grupo de investigación ECOSOL. Docente e investigadora de la Facultad de Ciencias Administrativas, Económicas y Contables, FUNLAM, Medellín, Colombia. Correo Electrónico: llopera@funlam.edu.co.

*** Administradora de la Fundación Universitaria Luis Amigó, Medellín, Colombia y magíster en Ética Social y Desarrollo Humano de la Universidad Alberto Hurtado de Chile, Santiago de Chile, Chile. Miembro del grupo de investigación ECOSOL, de la Facultad de Ciencias Administrativas, Económicas y Contables, FUNLAM, Medellín, Colombia. Correo electrónico: investigación@funlam.edu.co.

*** Zootecnista de la Universidad Nacional de Colombia, Medellín, Colombia; magíster en Agroforestería Tropical de CATIE, Centro Agronómico Tropical de Investigación y Enseñanza, Turrialba, Costa Rica; estudiante del doctorado de Ciencias Animales de la Universidad de Antioquia, Medellín, Colombia. Profesor asociado, Departamento de Producción Animal, Facultad de Ciencias Agropecuarias, Universidad Nacional de Colombia, sede Medellín, Colombia, Correo electrónico: ravelasquezv@unal.edu.co.
} 


\section{IS ORGANIC AGRICULTURE POSSIBLE IN MARINILLA?: IN BETWEEN THE RESOURCE CAPACITY AND POLITICAL WILL THERE IS A WAY}

ABSTRACT

The objective of this paper is to describe some socio-economic technical-agricultural characteristics of 28 production units of fruits and vegetables grouped in associations of the Marinilla municipalities in Antioquia Colombia. A methodology was used in the predominantly qualitative approach, supplemented with literature research and interviews with officials of the Municipal Unit for Technical Assistance in Agriculture and the Ministry of Agriculture. The most relevant result suggest that most of the units generate products for subsistence farmers and their families and for small volume sales for the local market. It also showed that most of the units do not develop organic production and in the case it been required for the sale of its products in the domestic market, is not given due to the importance of this type of production.

\section{KEY WORDS}

Associations, agriculture, agriculture and intrenational trade, food policy.

\section{JEL CLASSIFICATION}

D71, Q17, Q18, Q19

\section{CONTENT}

Introduction: 1. Organic production as substrate and sustainable human development, 2. Certification of organic production for a demanding market, 3. Conditions of organic agriculture in Marinilla: Certification opportunities 4. Conclusions, References.

\section{A AGRICULTURA ORGANICA É POSIVEL EM MARINILLA? ENTRE A CAPACIDADE DOS RECURSOS E A VONTADE POLITICA SE FAZ CAMINHO}

\section{RESUMO}

O objetivo deste artigo é descrever algumas características socioeconômicas e técnico agrícolas de 28 unidades de frutas e legumes agrupados em associações da municipalidade de Marinilla, Antioquia, Colômbia. Para isto, uma metodologia predominantemente quantitativa foi usada, a qual foi complementada por pesquisas bibliográficas e entrevistas com funcionários da Unidade Municipal de Assistência Técnica em Agricultura e do Ministério da Agricultura. O resultado mais relevante sugere que a maioria das unidades geram produtos para a subsistência dos agricultores e das suas famílias e para venda num pequeno volume para o mercado local. Também mostrou que a maioria das unidades não desenvolvem a produção orgânica, e no caso que foram impostas como requisito para a venda de seus produtos no mercado interno, não é dada a devida importância a este tipo de produção.

\section{PALAVRAS-CHAVE}

Associações, agricultura, agricultura e comércio internacional, a política alimentaria.

\section{CLASSIFICAÇÃO JEL} D71, Q17, Q18, Q19

\section{CONTEÚDO}

Introdução: 1. Produção orgânica como substrato e desenvolvimento humano sustentável, 2. Certificação da produção orgânica para um mercado exigente, 3. Condições da agricultura orgânica em Marinilla: oportunidades para a certificação 4. Conclusões, referências. 


\section{INTRODUCCIÓN}

A partir de un modelo de desarrollo que considera importante la sustentabilidad ambiental, según Prager y otros (2002), se resalta la necesidad de una producción agroecológica sana que no aumente los problemas del ecosistema y mejore el hábitat de la tierra y la salud humana. Se ha observado un auge y posicionamiento de los productos agroecológicos u orgánicos a partir del aumento de la demanda, por parte de países desarrollados, de productos alimenticios de buena calidad que no generen efectos nocivos y, en cambio, brinden mejor salud y bienestar a la población.

El tema de la producción agroecológica se ha relacionado con la explotación adecuada de los recursos naturales y con la producción agrícola que debe sustentarse en prácticas de calidad tradicionales, como la utilización de insumos orgánicos, y aspectos de connotación cultural relacionados con la responsabilidad en el consumo, para atender la salud humana. De esta manera, se ha generado una creciente preocupación por el desarrollo de sistemas de producción que posibiliten no solo la sostenibilidad económica, ambiental y política, sino también social.

En este artículo se presentan algunos resultados de la investigación, desarrollada por el grupo de investigación de Economía Solidaria (ECOSOL) de la Fundación Universitaria Luis Amigó (FUNLAM), en el municipio de Marinilla, municipio de la subregión del oriente antioqueño considerada como polo estratégico de desarrollo para el departamento de Antioquia (Colombia) debido, entre otros, a su ubicación geográfica, diversidad cultural, a la riqueza hídrica, biótica y paisajística, al potencial humano y a la presencia de infraestructura importante para el comercio internacional, en consonancia con las posibilidades de producción agrícola para atender la demanda interna y externa de la región.

De acuerdo con lo anterior el objetivo del artículo es describir algunas características socioeconómicas y técnico-agrícolas de 28 unidades de producción, consideradas como universo, de frutas y verduras agrupadas en asociaciones localizadas en el municipio de Marinilla.

Las unidades de producción que se incluyeron en el estudio fueron seleccionadas de acuerdo con criterios tales como el desarrollo de alguna actividad agrícola de carácter orgánico, la generación del producto para la venta y el hecho de pertenecer a alguna asociación de la región.

El estudio utiliza variables de naturaleza cuantitativa, registradas a través de un cuestionario de preguntas cerradas, tales como: estrato socioeconómico, actividad económica, productos cultivados, antigüedad de la parcela, destino de la producción, personas vinculadas al trabajo agrícola y prácticas agrícolas sustentadas en sistema de riego, conservación de la humedad del suelo, procedencia del agua para el riego, conservación de humedad del suelo, semillas para el cultivo y control de plagas. Dichas variables fueron medidas en la totalidad de las unidades de producción, para realizar análisis descriptivo de las principales variables que permitiera la exploración del tema de producción orgánica en Marinilla.

En general, el estudio evidenció que las unidades de producción pertenecían a campesinos, vinculados a las asociaciones, que en su mayoría pertenecen a estratos socioeconómicos bajos y que la posibilidad de generación de ingresos está vinculada con el empleo de su fuerza laboral en una empresa de producción agrícola, el asocio junto con su parcela a una organización, la producción en forma individual para aportar a la asociación o la producción individual para la venta en procura de los medios para la subsistencia.

Los agricultores que utilizaron alguna técnica relacionada con la agricultura orgánica no tenían incentivos ni de la empresa privada, ni del Estado, por lo que decidieron mezclar este tipo de agricultura con la convencional para reducir costos de producción y elevar el rendimiento de la tierra, que finalmente se agota y pierde fecundidad con esta práctica. 
En su mayoría, los agricultores manifestaron que en algunos casos les exigen estándares de calidad para la venta de los productos en el mercado interno, sin que se constituya en requisito permanente que les ponga restricciones y les obstaculice la venta o los obligue a adoptar normas de calidad, nacionales o internacionales.

El artículo pretende, además de presentar algunos hallazgos de la investigación, aportar elementos que aporten a la reflexión sobre un tema que en el fondo es bastante complejo y es el que tiene que ver con la sostenibilidad de la vida humana en el planeta y las condiciones de vida digna con equidad y justicia social que deben estar garantizadas para todos los seres humanos.

El artículo está organizado de la siguiente forma: en la primera parte se revisa el tema de la producción orgánica como aspecto esencial del desarrollo del ser humano, que se constituye en argumento para plantear que es una de las bases importantes sobre la que se conserva la vida del ecosistema del planeta. Con base en el anterior discurso, se deriva la propuesta de la soberanía alimentaria que según Galeano (2001), significa tener la responsabilidad y el compromiso de garantizar el futuro a las siguientes generaciones. La segunda parte sintetiza las formalidades de la certificación orgánica para abordar las exigencias de demanda en el mundo globalizado, que, sin embargo, se concretan casi en forma exclusiva en los países desarrollados. La tercera parte aborda algunas características socioeconómicas y técnico-agrícolas de las unidades de producción de frutas y verduras del municipio de Marinilla, con la evidencia empírica derivada de la investigación, sobre la condición de los agricultores para desarrollar la agricultura orgánica en el municipio. Por último, se concluye sobre las intenciones de generar una agricultura que esté en la base del cuidado de la salud humana y la vida de la tierra, pero que no alcanza a concretarse por las condiciones reales en que viven los agricultores que cuidan la base alimentaria de las regiones.

\section{LA PRODUCCIÓN ORGÁNICA COMO FUNDAMENTO DEL DESARROLLO HUMANO Y SUSTENTABLE}

Según Polanco y otros (2005), la preocupación por el mantenimiento y la conservación del ecosistema y la capacidad agrícola que posibilite la producción alimentaria para sostener a las futuras generaciones, dio origen al concepto de "Desarrollo Sustentable", el cual quedó expresado en el informe Brundtland (ONU, 1987b).

Dicho informe fue realizado y presentado por la ONU (1987a), en la Comisión Mundial sobre Ambiente y Desarrollo, y ha dado origen a varias interpretaciones y derivaciones que fueron incorporándose a los conceptos de bienestar y calidad de vida, tales como la sustentabilidad ecológica, económica, social, política y cultural, entre otros. A partir de la promoción de este paradigma en el discurso del desarrollo, se intentó dar solución a los problemas calificados como de mayor trascendencia y de difícil aplazamiento, tales como la pobreza y la seguridad alimentaria. Como se comprende, la ONU es también uno de los más importantes promotores del desarrollo sostenible o sustentable.

Polanco y otros (2005) sostienen que en los últimos años el concepto de sostenibilidad se ha centrado en objetivos de desarrollo humano, y que ha adquirido significados cada vez más precisos, como el eco-desarrollo y desarrollo sostenible, y ha pasado de ser una preocupación para convertirse en un compromiso de política en la planeación y puesta en marcha de modelos productivos de desarrollo. De este modo, también ha derivado en otras propuestas tales como la sustentabilidad ecológica, sustentabilidad ambiental, sustentabilidad social y sustentabilidad política.

De acuerdo con Polanco y otros (2005):

La sustentabilidad ecológica, se refiere a la base física del proceso de crecimiento y al mantenimiento del stock de recursos naturales incorporados a las actividades productivas. La sustentabilidad ambiental hace referencia a 
la capacidad de la naturaleza para absorber y recomponerse de las agresiones al medio. La sustentabilidad social tiene por objeto el mejoramiento de la calidad de vida de la población. La sustentabilidad política se encuentra estrechamente vinculada al proceso de construcción de la ciudadanía, y todas en su interdependencia buscan garantizar la incorporación plena de las personas al proceso de desarrollo.

El concepto de sostenibilidad también formó parte en la ONU (1992) de la Declaración de Río de Janeiro, elaborada desde la Cumbre para la Tierra, como parte de las estrategias mundiales para el manejo del entorno ambiental, en articulación con el desarrollo económico que debe garantizar la sostenibilidad del ecosistema mundial.

\subsection{La agricultura en la base de la sostenibilidad del ecosistema}

Etimológicamente, la expresión agricultura hace una reminiscencia a una cultura del campo y a una idea de que es aquello que emerge como origen de todo fenómeno natural, que está en la base para construir el devenir o el futuro. En otras palabras, remite al origen, unido al concepto de la naturaleza que fue asimilado hace miles de años por el ser humano.

Según Rosas (2003), la agricultura es una de las actividades más antiguas de la humanidad, se constituye como proceso natural que depende de un ecosistema en el que un variado y extenso conjunto de factores (que aún continúan en proceso de descubrimiento y más con la compleja relación que se genera entre ellos), influyen para determinar el resultado de una actividad agrícola. Ese conjunto de factores, tales como la energía, los ciclos de nutrientes y del agua, el estado del suelo, entre otros, interactúan a través procesos complejos e interdependientes, de tal manera que no se puede afectar un solo factor o elemento de los intervinientes, sin modificar la totalidad del conjunto o universo.

De acuerdo con Rosas (2003), la agricultura, a diferencia de la industria, es un proceso natural que depende de un ecosistema y tiene una relación directa con el medioambiente, en el que se produce una interrelación entre la naturaleza y el trabajo del ser humano. En este sentido, puede considerarse un agro ecosistema en el que debe respetarse su biota para la reproducción del mismo, al contrario de lo que ocurre en la agricultura convencional, en la que el desequilibrio de la biota contamina el componente abiótico, por la intervención de los agroquímicos, lo que afecta el ecosistema.

En la actualidad, cobra fuerza la demanda de producción agrícola, en condiciones particulares de inocuidad para la salud humana y el mantenimiento de los ecosistemas para el sostenimiento de la vida del planeta. De esta manera, los productos orgánicos son alimentos que ayudan a conservar la salud, mientras que la agricultura basada en agroquímicos produce alimentos incompletos, que no tienen todas las vitaminas y elementos esenciales, pero en cambio, sí contienen residuos de pesticidas con moléculas químicas tóxicas.

De acuerdo con Rosas (2003), la agricultura orgánica o ecológica es toda una filosofía y doctrina para la equidad, el respeto a la naturaleza, la sostenibilidad de las generaciones presentes y futuras, que propende por la conservación del planeta tierra y todos los seres vivos.

\subsection{La agricultura orgánica como base natural de la sustentabilidad ambiental}

Según Rosas (2003), el concepto técnico y la aplicación de la agricultura orgánica surgen en la Gran Bretaña después de la Segunda Guerra Mundial, fundamentados en la protección del suelo, la fertilización orgánica y la conservación de la biodiversidad, unidos también al concepto de verificable, lo cual permite que se especifique en un conjunto de normas internacionales reconocidas y la creación de instituciones que certifican su cumplimiento.

De este modo, la agricultura orgánica se concreta en un acumulado de prácticas, articuladas a una producción agrícola sostenible, en relación 
con el medioambiente, los recursos naturales, la biodiversidad y el cuidado de la vida y la salud del ser humano a través de la alimentación sana. Además, se corresponde con la restricción de la utilización de insumos externos como los plaguicidas y fertilizantes de síntesis química o semillas transgénicas (organismos modificados genéticamente-OMG) a cambio de realizar las prácticas fitosanitarias y de producción a partir de procesos y controles naturales o biológicos para obtener producciones limpias, de mayor calidad nutricional, inocuas que no generen problemas a la salud humana y preserven el entorno o ecosistema.

De acuerdo con Rosas (2003), las prácticas de agricultura orgánica están soportadas en la sabiduría y conocimiento ancestrales de las comunidades rurales, relacionadas con la conservación de todos los elementos de la naturaleza. Sostiene que estas prácticas resurgen en momentos en que la agricultura convencional o comercial llega a estados insostenibles en cuanto a disminución de la productividad y a generación de efectos negativos en el medioambiente con resultados nocivos en el ecosistema mundial.

De este modo, la agricultura orgánica genera un discurso articulado que proviene de algunas propuestas de organismos internacionales intervinientes en la industria alimentaria en el mundo, tales como la Organización de la Naciones Unidas para la Agricultura y la Alimentación, FAO (2003), La Federación Internacional de Movimientos de Agricultura Orgánica, IFOAM (2000) y el Programa Especial para la Seguridad Alimentaria, PESA (FAO, 2003), entre otros. De allí se deriva la creación en 1963, por la FAO, de la Comisión Codex Alimentarius para la promoción de normas alimentarias relacionadas con métodos agronómicos, biológicos y mecánicos, en oposición a la utilización de materiales sintéticos, en la perspectiva de atender el cuidado de la salud humana.

En este sentido, IFOAM (2000) define la agricultura orgánica como el conjunto de los sistemas agrícolas que promuevan la producción sana y segu- ra de alimentos, basados en la fertilidad propia del suelo, el respeto por las exigencias y capacidades naturales de las plantas, los animales y el paisaje. Desde esta perspectiva, se comprende por qué se espera que sean las leyes de la naturaleza las que incrementen tanto los rendimientos, como la resistencia de los cultivos, lo cual reduce las necesidades de aportes externos.

En sentido general, puede plantearse que la producción agrícola orgánica asume una relación con la tierra y con la naturaleza que requiere de un pensamiento diferente para integrar las dimensiones social, ambiental, económica y cultural relevantes en el contexto y medio rurales; por lo tanto, no debe ser analizada solo en términos de costos y ganancia, sino que debe articular otros aspectos que se vinculan con la seguridad alimentaria y la vida del planeta tierra en las diferentes dimensiones.

\subsection{La agricultura orgánica y la seguridad alimentaria en la base de la sustentabilidad social y política}

Según Thompson y Metz (1999), el desarrollo sustentable no se agota en la sostenibilidad de los recursos naturales y el cuidado ambiental, sino que también incorpora otros aspectos que pertenecen al ámbito de lo social y político, tales como la autonomía para la producción de alimentos y el acceso a estos en condiciones favorables. En este sentido, la FAO (1996) presenta como objetivos de la seguridad alimentaria: garantizar a todos los seres humanos el acceso físico y económico a los elementos básicos necesarios y posibilitar la oferta de alimentos a precios razonables; en tal sentido, una persona tiene seguridad alimentaria cuando el consumo responsable es siempre mayor que las necesidades fisiológicas. De este modo, el consumo se determina por el derecho de la persona a los recursos alimentarios que deben estar disponibles en el hogar.

Así, de acuerdo con Thompson y Metz (1999), se puede presentar inseguridad alimentaria cuando 
un país no puede producir o importar abastos suficientes para satisfacer la demanda del mercado interno, lo cual genera el alza de los precios, que deja expuesto a un número creciente de hogares a sufrir los rigores de esta inseguridad; por consiguiente, también se requiere revisar las disposiciones de política económica de tal manera que se garantice la estabilidad económica del país que padezca estos problemas.

De acuerdo con Sánchez (2005), la seguridad alimentaria es una medida de bienestar de carácter universal, que incluye el ámbito de la vida tanto familiar, como individual, y que también se relaciona con la soberanía alimentaria, entendida como el derecho de cada nación de mantener y desarrollar su capacidad para producir los alimentos básicos y la capacidad de procurárselos en correspondencia con las especificidades sociales, económicas, ambientales y culturales, con respeto por la diversidad productiva y cultural, y atención al cuidado de los recursos naturales.

Según Mejía (2003), la soberanía alimentaria se define como proyecto político, en la medida en que se establezca la garantía de proporcionar a la población los abastos corrientes necesarios, lo cual significa que la sociedad debe enfrentar la condición de la propia supervivencia, en forma particular cuando define modalidades de uso y posesión de la tierra apropiados para el logro de la seguridad y la soberanía alimentaria, con adherencia al proyecto político de soberanía nacional, desde donde se define la independencia del país para procurar los alimentos necesarios y suficientes para la sostenibilidad de sus habitantes.

En tal sentido, según Sánchez, (2005), la soberanía alimentaria, en su propósito fundamental, contribuye con la reafirmación del derecho humano de acceder a los alimentos, como sustentadores de la vida y perfila la necesidad de una reforma agraria que garantice a las familias campesinas, productoras de alimentos por tradición, la tenencia y el control de la tierra en la que trabajan y la devolución de los territorios a los grupos humanos que tradicionalmente los han tenido y han sufrido despojo.

Plantea Forero (2003), que la seguridad alimentaria, sustentada en la agricultura familiar es, tal vez, el único sostenimiento del que disponen muchos hogares pobres. Es conocido que la pobreza es un fenómeno que golpea con mayor fuerza en el ámbito rural, y ante condiciones desfavorables se genera un círculo vicioso en el que la producción de alimentos depende de un sector con altas restricciones económicas y en el que paradójicamente está la mayor despensa de nutrientes y vida del planeta.

Según la FAO (1996) la seguridad alimentaria debería establecerse como prioridad máxima de la Organización, y de este modo, concentrarse en programas que buscarían el logro de incrementos en la producción de alimentos, la mejoría en la estabilidad de las reservas y la creación de empleos rurales para facilitar así la disponibilidad general de los suministros; también debería ponerse en marcha el Programa Especial para la Seguridad Alimentaria en los países de bajos ingresos y con déficit de alimentos.

Además, la Cumbre Mundial sobre la Alimentación (1996) suscribió el anterior planteamiento, lo cual exige un esfuerzo conjunto en todos los campos para elevar la producción de alimentos y lograr que 86 países tengan mayor acceso a estos, con el propósito de reducir a la mitad, hacia el 2015, la cantidad de personas desnutridas del mundo. El plan de acción adoptado por la Cumbre concluyó que para reducir el hambre era necesario asegurar condiciones propicias, mejorar la elaboración y el acceso a los productos, reforzar la función del comercio, hacerse cargo de los casos de desastre e invertir en la seguridad alimentaria.

Según Robledo (2003) el concepto seguridad alimentaria no solo se refiere al problema de asegurar la existencia y la producción de los alimentos de un país, sino también de garantizar que lleguen a la población más pobre; esta es la principal razón, la que supedita a las restantes por importantes que 
sean, que justifica el gasto que hacen en subsidios a su agro los 29 países más ricos de la Tierra, cerca de 370 mil millones de dólares al año por este concepto, cifra que crece de manera ininterrumpida desde hace décadas, tanto que en la correspondiente a la década de los noventa mostró un incremento de 50 mil millones.

Por su parte, a los países en desarrollo se les presentan varios compromisos: el de generar alimentos suficientes para cubrir la necesidades nutricionales de la población con precario acceso, con atención primordial a la más pobre; producirlos en condiciones orgánicas para atender el cuidado de la salud humana y del medioambiente y procurar alcanzar los estándares de calidad exigidos en el mercado mundial, que posibiliten desarrollar las ventajas competitivas necesarias para situar la región y las localidades en el mercado mundial como productoras de alimentos orgánicos.

Como se evidencia, la producción orgánica es un discurso siempre vigente, cuando de soberanía alimentaria se trate y en relación con las posibilidades de desarrollo humano sostenible, en realidades concretas y específicas de los grupos humanos, y dentro de esta perspectiva con la potencialidad para inscribirse de manera importante dentro de las exigencias y requisitos presentes en la demanda mundial de alimentos y su normativa.

\section{LA CERTIFICACIÓN DE LA PRODUCCIÓN ORGÁNICA PARA UN MERCADO EXIGENTE}

Recientemente se ha consolidado un movi miento mundial en torno a la preocupación por las características del consumo alimentario: se interrogan por el origen de los productos, de cómo se elaboran y comercializan y cuál es su contenido para proteger la salud y su seguridad.

La agricultura orgánica cumple el doble propósito de posibilitar el cuidado de la salud humana y del medioambiente, y de constituirse en opción de vida para los consumidores, por el uso de abonos orgánicos, con lo cual se superan las formas de producción convencionales, de producción agroquímica, que han contribuido al deterioro de los recursos naturales y la vida del planeta.

En Colombia, cada año aumenta el número de hectáreas limpias que se dedican al cultivo orgánico para complacer a los consumidores, tanto colombianos como de otros países, que están en capacidad de pagar precios más altos por proteger su salud. Sin embargo, la producción no abastece la demanda mundial por alimentos. Países de Europa, Asia y América del Norte demandan, cada vez más, este tipo de productos y establecen, como prioridad, que sean de carácter ecológico.

Según Espinoza (2004), la creciente preocupación de muchos consumidores de los países desarrollados por la protección del medioambiente y por el cuidado de la salud, unida a la frecuente identificación de enfermedades transmitidas por alimentos (ETA), han reducido la confianza en los sistemas convencionales de producción de alimentos y, a la vez, han generado un incremento en la demanda de productos de origen ecológico desde los primeros años de la década de los ochenta.

Según la FAO y la Organización Mundial de la Salud, OMS (2004), entre los desafíos que comprometen a las autoridades para ejercer control y regulación se encuentran la creciente carga de las enfermedades transmitidas por los alimentos y aparición de nuevos peligros relacionados con estos productos, los cambios rápidos en las tecnologías de producción, la elaboración y comercialización , el desarrollo de sistemas de control de base científica orientados a la protección del consumidor, el comercio internacional y la necesidad de armonización de las normas de inocuidad y calidad de los alimentos, la creciente conciencia de los consumidores sobre la calidad de los productos y la mayor demanda de información por parte de estos. La responsabilidad máxima la constituye el establecimiento de leyes de protección al consumidor frente a alimentos peligrosos, impuros, con presentación fraudulenta 
y que no correspondan a la naturaleza, sustancia o calidad exigidas por el comprador.

De acuerdo con el Ministerio de Agricultura y Desarrollo Rural (2002), en Colombia la Resolución 0074 del mismo año, es la que define el sistema de producción ecológica que enmarca la elaboración de alimentos sanos y seguros, desde el punto de vista ambiental, social y económico, con lo cual se busca reducir la cantidad de pesticidas per cápita y hacer control de los peligros transmitidos por el consumo de estos productos. La resolución establece el reglamento para la producción primaria, procesamiento, empacado, etiquetado, almacenamiento, certificación, importación y comercialización de productos agropecuarios de carácter ecológico.

De acuerdo con Espinoza (2004), el consumo de productos ecológicos en la Unión Europea ha crecido a una tasa promedio del $25 \%$ en los últimos 10 años. Alemania sería el responsable de la mitad del consumo total, hecho que lo convierte en el principal mercado, seguido por Francia. Según Pons y Sivardiére (2002), la Unión Europea estableció en 1991 la legislación internacional en Agricultura Orgánica (EEC No. 2092/91) que regula a todos los países miembros y a todos aquellos países que deseen exportar productos ecológicos a la UE. Según consta en FAO (2001), Estados Unidos estableció su Ley Federal en 1993 pero su implementación y reglamentación entró en vigencia a partir del año 2002, por su parte en Japón, a partir del $1^{\circ}$ de abril del 2001, entró a regir la Normativa para Producciones Orgánicas del Ministerio de Agricultura Forestación y Pesca-MAFF que exige el etiquetado con el sello de Japanese Agricultural Estandars, JAS, en los productos agrícolas orgánicos con destino a la comercialización.

Según Saborío y Delgado (2006), la certificación es lo que posibilita que un vendedor puede asegurar, a quienes compran sus productos, que son generados bajo normas de producción orgánica reconocidas, tanto en el ámbito nacional como internacional. La certificación marca la diferencia entre la comercialización de un producto orgánico y otro cultivado en forma convencional.

De este modo, la certificación para la producción orgánica es el procedimiento mediante el cual se garantiza que un determinado producto animal o vegetal, los equipos y el proceso de producción, cumplen con las normas de un organismo regulador orgánico, sin dañar el medioambiente. De acuerdo con Saborío y Delgado (2006), para certificarse no se requiere ser miembro de una Organización Orgánica y puede obtenerse el sello de certificación por tres años, que puede prorrogarse siempre y cuando el titular mantenga su sistema productivo conforme con los requisitos establecidos.

Así, la certificación es una herramienta para la comercialización transparente de los productos orgánicos, le da al consumidor la confianza de adquirir un producto alimenticio, inocuo para su salud, en la misma forma que el productor demuestra que su producto es diferente a los convencionales.

Según FAO (2003) y FAO y OMS (2004), el fundamento lo proporciona el Código Alimentario que es un conjunto de normas internacionales, reguladoras de la producción de los principales productos de esta naturaleza -las normas se adoptan de manera voluntaria para cuidar la salud de los consumidores a través de la protección de alimentos genuinos, debidamente etiquetados y no adulterados--, y de códigos de prácticas, directrices y recomendaciones que buscan la seguridad de los consumidores.

De acuerdo con FAO y OMS (2004), a la Comisión del Codex Alimentario le corresponde formular propuestas a los directores generales de estos organismos, y figura como ente de consulta, en todas las cuestiones relativas a la ejecución del Programa Conjunto FAO/OMS sobre Normas Alimentarias relacionadas con la protección de la salud de los consumidores, el aseguramiento de prácticas equitativas en el comercio de los alimentos y la promoción de la coordinación de todos los trabajos sobre normas alimentarias, que puedan emprender 
las organizaciones internacionales gubernamentales y no gubernamentales.

Como lo plantean Saborío y Delgado (2006), las normas de producción orgánica son bastante parecidas en todo el mundo, en cuanto a los principios generales, y deben ser certificadas por una organización competente, lo cual les confiere confiabilidad para que comprometan la imparcialidad e independencia de sus directores a la hora de tomar decisiones sobre el otorgamiento o no de una certificación.

En el ámbito internacional, la Federación Internacional de Movimientos de la Agricultura Orgánica, IFOAM (2000) es la encargada de la producción de normas básicas de acreditación para la certificación: establece los requisitos para la agricultura orgánica y acredita a los organismos certificadores que revisan el cumplimiento de dichas normas junto con la entidad competente de cada país.

Colombia no es ajena al tema y en consonancia con la tendencia mundial de la demanda por alimentos producidos orgánicamente, creó los distritos agrarios. En efecto, ante las condiciones internas del país, las preocupaciones por la subsistencia que agobian a cada comunidad en concreto y las posibilidades reales que se inscriben en contextos locales, en los que se manifiestan las exigencias internacionales y lo compromisos institucionales, se idearon los distritos agrarios que deben orientarse a fomentar el desarrollo rural, la protección de la economía campesina y a favorecer la producción de alimentos que sea sostenible y que contribuyen a la conservación de los recursos naturales.

En este contexto, en Marinilla se creó el Distrito Agrario para fortalecer los aspectos mencionados, en razón de su vocación agrícola y las posibilidades de articulación con el mercado local, nacional e internacional; sin embargo, cabe interrogarse por las condiciones concretas de las subregiones y del municipio, en particular, para inscribirse como realidad local en el concierto mundial y más aún como posibilidad agroalimentaria de origen orgánico.

\section{CONDICIONES DE LA AGRICULTURA ORGÁNICA EN MARINILLA: POSIBILIDADES PARA CERTIFICACIÓN}

Marinilla es un municipio de la subregión del Oriente antioqueño, tiene un área de 118 kilómetros cuadrados, con una población de aproximadamente 40.075 habitantes; la principal actividad económica de Marinilla ha sido históricamente la agropecuaria y se ha considerado, junto con otros municipios de la subregión, una despensa del Valle de Aburrá.

Según Suaza (2008), la producción agrícola del municipio ha estado destinada al abastecimiento de las unidades domésticas. A medida que el desarrollo del siglo XX incentiva el trabajo de la tierra y se incrementan los niveles de productividad, unidos al avance en productos químicos como fungicidas y fertilizantes, se inicia una etapa de posibilidades de acceso a los mercados regionales, que induce la transformación de las economías familiares, domésticas, las cuales adoptan un sistema de producción agrícola intensivo, dependiente de agroquímicos y sustentado en una explotación de economía campesina de minifundio, en la que hay una utilización máxima de la fuerza de trabajo familiar. Este modelo de explotación solo permite satisfacer las necesidades básicas de la familia campesina, sin posibilidades de generar excedentes económicos que sustenten un proceso de acumulación y hagan de la actividad agrícola una empresa familiar.

De acuerdo con Suaza (2008), en los últimos veinte años, el municipio ha crecido en la diversificación de la producción agrícola: pasó de solo tres productos cultivados tradicionalmente (papa, maíz y fríjol), a la oferta de una variedad de hortalizas tales como: repollo, zanahoria, remolacha, brócoli, coliflor, lechuga, pimentón, tomate y arracacha, entre otros, con cierta estabilidad en su ritmo de crecimiento, debido a que encuentran un amplio mercado en los municipios del Valle de Aburrá y en los de otros departamentos, principalmente en los de la Costa Atlántica. 
La propuesta de diversificación agrícola provino de las recomendaciones de la Unidad Municipal de Asistencia Técnica Agropecuaria, UMATA, fundamentada en un censo realizado en las veredas en el año 1993, que le permitió determinar los problemas y las necesidades del sector rural, para basar los respectivos diagnósticos de los programas agrícola, ambiental, de comercialización y organización comunitaria. A partir de allí, la UMATA impulsó un esquema de producción intensivo en la utilización de agroquímicos, con la idea de lograr un aumento en el rendimiento y la productividad y una disminución de los costos, en el marco de un modelo de revolución verde.

Según Suaza (2008), dicho modelo incluía metodologías basadas en comparaciones, demostraciones y experimentos que servían para introducir la propuesta tecnológica de producción, presentada con mayores ventajas frente a las que se sustentaban en la práctica tradicional realizada por los agricultores, lo cual derivó en la obtención de distintas variedades adaptables para ser promovidas en el municipio, tales como: el brócoli, la coliflor blanca, el zuquini, el apio y el rábano, entre otros. La moderna tecnología, que traía consigo mecanización, fertilización química y agro tóxica, termina por ser aplicada en el Oriente Antioqueño, y en especial en Marinilla.

Según Suaza (2008), la modificación del estilo de producción campesino, históricamente ensayado y practicado como un modelo de producción autónomo, con la revolución verde se alteró, porque derivó en la utilización de insumos externos y, contrario a lo esperado, en incrementos en los costos de producción. De manera simultánea, comenzó a desdibujarse el paisaje, y a deteriorase en forma progresiva el suelo, el agua y los bosques.

Más tarde, como estrategia orientada a resolver los problemas generados por las prácticas agrícolas de la revolución verde, aparece la SAYA (Secretaría de Agricultura y Medio Ambiente) que interviene con propuestas de desarrollo rural participativo para involucrar a los campesinos en el diseño e implementación de instrumentos que incidieran en el mejoramiento de las condiciones de vida. Según Suaza (2008) un ejemplo de esta etapa de transición fue la estrategia de capacitación continua bajo la metodología de escuela campesina, que concebía la educación como un proceso continuo y participativo, e incluía estrategias pedagógicas que estuvieran acordes con la necesidad de hacer confluir, tanto los intereses relacionados con la producción y la generación de ingresos, como los de conservación, educación y la organización de los agricultores.

En el proceso de cambio de estrategia para responder a la demanda de países desarrollados, por productos de origen orgánico, se continúa en el propósito de establecer estrategias de ampliación de la oferta alimentaria que permitan el abastecimiento del mercado local y regional; en este sentido, de acuerdo con Alzate y otros (1999), se concretó la creación de un Distrito Agrario Subregional, mediante el "Programa de Creación, Declaratoria, Manejo y Administración de un distrito Agrario en el municipio de Marinilla-Antioquia"; luego, la Administración Municipal incluyó el Distrito Agrario dentro de su Plan de Ordenamiento Territorial dispuesto por la Ley 388 de 1997, y en 1999 se crea, por Acuerdo del Concejo Municipal de Marinilla, el Distrito Agrario de Marinilla, según Rodríguez (2000), para impulsar el desarrollo integral de la actividad agropecuaria , desde una perspectiva que le otorgó posibilidades de conservación a la región, con su vocación productiva y la adopción de reglamentaciones para el manejo de varias "Zonas de Reserva Agrícola", con fundamento en principios constitucionales, jurídicos, tecnológicos, ambientales y socioeconómicos.

En la actualidad, algunas parcelas trabajan bajo el esquema de buenas prácticas agrícolas, BPA, conjuntamente con el modelo convencional aprendido en los años de la revolución verde, con la intención de orientar la producción hacia la agricultura orgánica, con recomendaciones de la UMATA y la SAYA, que proponen una estrategia de 
asociación, para que los productores se organicen y logren la adaptación de prácticas de producción de este tipo y puedan negociar en forma directa los productos.

La investigación se desarrolló en las siguientes veredas del municipio de Marinilla: La Inmaculada, Aldana, Alto del Mercado, Asunción, Cascajo, Chocho Mayo, Concentración Montañita, Cristo Rey, El Chagualo, El Pozo, El Rosario, El Socorro, Gaviria, La Esmeralda, La Esperanza, La Granja Chagualo, La Inmaculada, La Plata, La Primavera, Las Mercedes, Llanadas, La Peña, Pantanillo, Porvenir, Salto Arriba, Salto Abajo, San Bosco, San José y Santa Cruz.

De las anteriores veredas solo en San José, Salto Arriba, Salto Abajo, Alto del Mercado, Cristo Rey y Llanadas hay unidades de producción que practican la agricultura orgánica. Los agricultores de estas unidades se encuentran asociados a Agropaisa, Asocampo y Asofrumar, organizaciones que se interesan por los temas relacionados con el desarrollo de la agricultura orgánica.

No obstante, se puede decir que en Marinilla las asociaciones han sido las que han promovido las actividades agrícolas tradicionales y las de origen orgánico, las búsquedas de capacitaciones y los vínculos con la Administración municipal o con otras asociaciones.

Las asociaciones que procuraron organizar su producción agrícola como orgánica, además de su carácter ambientalista, han demostrado conocimiento respecto a sus propias prácticas, si son orgánicas o no, pero el 66\% de los consultados desconocían que Marinilla fuera distrito agrario y lo que esto significaba. De igual modo, han trabajado por generar una conciencia entre los productores para que practiquen la agricultura orgánica; sin embargo, les falta mayor aprendizaje, han sabido cuál es el camino y han sido conscientes de que el problema no radica en que el agricultor no sepa trabajar la tierra, sino que la falta de ingresos, el deterioro del medioambiente y la dificultad para marginarse del uso de los químicos-sintéticos, comercializados en los mercados locales y nacionales les obstaculizan la adopción de este tipo de producción en su totalidad.

De los agricultores de Marinilla encuestados, el $72 \%$ se ubicaba en el estrato dos y el 19\% en estrato tres. La unidad económica en un 56\% de los casos era de propiedad familiar, lo que justificaba por qué en parte se dedica primero a generar la sostenibilidad alimentaria de los parientes y luego a la producción para la venta, orientada por las asociaciones. Los agricultores, jefes de hogar, deben sostener en su mayoría a estudiantes, adultos mayores, personas con discapacidad y desempleados. Cabe anotar que la producción para el sustento familiar genera mayor vulnerabilidad en estos grupos poblacionales porque la seguridad alimentaria depende de la capacidad de asociación y de la disponibilidad de la parcela agrícola.

Las actividades agrícolas de las unidades económicas ocupaban a las propias familias, entre 1 y 4 personas, en su mayoría mujeres, muchas veces sin ningún tipo de contraprestación, solo la motivación de trabajar para procurarse la subsistencia por pertenecer a ese núcleo, sin que haya percepción del sobre trabajo en la producción, sino en relación con las ocupaciones domésticas; el 29\% de los encuestados recibían como pago por su actividad una cantidad de dinero, combinada con otras contraprestaciones en especie, en condiciones por debajo del salario mínimo legal vigente (461.500, Decreto 4965/2007), o no estaba calculado.

La principal actividad productiva desarrollada por los agricultores encuestados era el cultivo de frutas y verduras para la economía familiar, en un 69\% dedicaban su actividad a la producción de maíz, repollo, zanahoria, papa criolla, chócolo, frijol, brócoli, mora y arveja, entre otros, siempre con la esperanza de producir para el mercado regional o el externo, por lo que las asesorías de la UMATA representaban una promesa que les permitiría, en el mediano plazo, la aplicación de prácticas adecuadas según estándares internacionales que 
los calificarían para el mercado internacional, acogiéndose a las directrices de la entidad que sugiere las características de la producción, ya sea para explotar la parcela con destino al mercado interno de acuerdo con las características del suelo o para el mercado externo.

Las parcelas de Marinilla reconocidas por la investigación tenían una antigüedad superior a 5 años, lo cual justificaba la necesidad de desaprender las antiguas prácticas agrícolas con químicos, dejadas por la cultura de la revolución verde, antes de asumir completamente una de carácter agroecológica, con acompañamiento por parte del Estado, por ejemplo, con políticas de subsidios como lo hacen en países que han acogido la producción orgánica, pues la evidencia es que solo el $11 \%$ de los agricultores recibían algún apoyo y el resto subsistían gracias a sus propias capacidades de producción.

Los agricultores reconocieron que su tradición agrícola se mantenía en la cultura de la revolución verde, que no significa prácticas agroecológicas; aunque conocían bien sus cultivos no aplicaban sello de calidad, y manifestaron que en algunos casos les exigían estos estándares para la venta de los productos en el mercado interno, pero sin constituir obstáculo para la venta en la localidad.

El trabajo de las asociaciones ha sido específico en enseñar prácticas agrícolas para la producción orgánica, que debe estar certificada por una norma internacional que permita la actividad para mercados internacionales. Según Clements (1997), la norma ISO verde (ISO 14.000), da una estructura o pauta para administrar un sistema que puede ser llamado de gerencia ambiental. En tal sentido, ¿Se podría pedir a los agricultores de pequeña escala, del municipio de Marinilla, organizados o no, a las asociaciones o gremios, cumplir con requisitos de estándar internacional para lograr un sistema de gerencia integral (término que no es utilizado en el lenguaje del productor) y así exportar y competir según estándares del mercado internacional?
Algunas instituciones y personas involucradas con las actividades agrícolas de Marinilla, saben que deben empezar por la descontaminación de la tierra y principalmente, por concienciar a los productores y a los consumidores, en general, de las bondades de la agricultura orgánica para el cuidado de la salud humana y la vida de la tierra y que estas posibilidades se logran a través de la organización comunitaria. En tal sentido, cabe educar en aspectos que relacionen los sistemas vivos que es necesario reconocer para cuidar, la identificación del municipio como territorio en el que se aplica la agricultura orgánica y la dinámica de los recursos naturales en su vínculo con el entorno, a partir del trabajo en común y las formas de organización comunitaria con el propósito de definir el trabajo consciente y planificado para que se concreten mejores posibilidades de cuidado para la salud humana y mayores ingresos a la economía familiar. Tal como lo expresa Jaramillo (2008), funcionario de la Secretaría de Agricultura de Marinilla: "Entonces, a través de las organizaciones lo que se busca también es acabar con la intermediación y (enseñar) y hacer que ellos (los productores agrícolas) dejen de ser dependientes de (los) agentes externos y enseñarles que lo produzcan ahí, en la misma tierra, de lo contrario, no hacemos nada".

Desde esta perspectiva, se comprende que, de acuerdo con Jaramillo (2008), aún "hay camino por recorrer, (lo cual significa) asumir con fuerza los compromisos y responsabilidades que supone el sostenimiento alimentario de la comunidad". Marinilla, por ser un municipio con una gran variedad de hortalizas, frutas y verduras, resulta muy llamativo, no solo para los comercializadores de abonos, sino también para quienes pretenden entrar en el negocio de la exportación, y las políticas que adopta el Estado para apoyar al pequeño productor agropecuario y disminuir el deterioro del medioambiente no son suficientes, y aun se requiere que la agricultura de Marinilla se vuelva en su totalidad orgánica y libre de químicos, de tal manera que se generen las condiciones para 
la sostenibilidad alimentaria de la región y la producción para la exportación..

Cabe preguntarse si las asociaciones, en general, el sector privado y los entes gubernamentales tienen la convicción y la voluntad de procurar el cuidado de la salud y del medioambiente para tener la posibilidad de que la población pueda vivir un poco mejor y para dejar algo a las futuras generaciones, tal como lo plantea el informe Brundtland (ONO, 1987) ¿Qué se hace al respecto?

Finalmente, es preciso recordar que la preocupación por la salud humana, relacionada con la producción orgánica, proviene de la conciencia sobre la relación que hay entre los alimentos, la nutrición y la condición saludable de las personas y que son los pequeños agricultores y su vínculo con la tierra, los que proveen de alimentos a la región. En este sentido, todos saben del círculo virtuoso que se genera si hay apoyo para los pequeños productores: favorece su ingreso y el de las familias, se mejora su acceso a los servicios de educación y salud, y por esta vía su capacidad productiva, y si se le adiciona la capacidad de organización y de asociación, se multiplicarán las posibilidades de ampliación de la oferta alimentaria para la región y para la exportación y desde allí, la soberanía alimentaria es un imperativo.

\section{CONCLUSIONES}

El estudio sobre características socioeconómicas y técnico-agrícolas de 28 unidades de producción de frutas y verduras agrupadas en asociaciones del municipio de Marinilla deja en evidencia la problemática que hay en la relación de las posibilidades de producción agrícola y su vínculo con la sostenibilidad del ecosistema. Desde esta perspectiva, se presenta el tema específico de la agricultura orgánica en un municipio del oriente antioqueño, en el marco de la demanda mundial por alimentos de origen orgánico y ante las exigencias internacionales de certificación de este tipo de producción. El tema reviste trascendencia por las implicaciones de la agricultura como sustento de cualquier sociedad que requiere concretar las posibilidades de la oferta alimentaria para las comunidades territoriales, las regiones y el mercado mundial.

Dentro de esta lógica, se crearon los distritos agrarios en la Valle de San Nicolás, oriente antioqueño, y en específico en Marinilla como experiencia piloto, para definir el fortalecimiento de los municipios de la región, que supone ampliar la capacidad de los recursos para responder a la demanda interna de las regiones y la necesidad mundial de producción de alimentos de origen orgánico, en un contexto en el que cada vez es más importante la relación entre la producción agrícola, el cuidado de la salud humana y la protección del medioambiente.

Sin embargo, es contradictoria esta tendencia en la lógica interna del sistema socioeconómico predominante que, de acuerdo con Blunt (1998), mantiene los principios de eficiencia y agilidad en los procesos productivos al costo monetario más bajo posible, sin medir las consecuencias de los hechos económicos y de los efectos negativos sobre la supervivencia del ecosistema. Según este planteamiento, el modelo de producción vigente es insostenible en los esquemas de producción y de consumo, debido a que se apoya en formas agresivas de transformación de los recursos naturales para obtener rentabilidad, sin importar el costo social y las consecuencias negativas sobre el ambiente.

De igual modo, el consumismo soslaya las preguntas relacionadas con el origen de los productos, la forma como se producen, la clase de insumos utilizados y el destino final de los residuos considerados inútiles, lo que representa un riesgo potencial muy elevado para la salud humana y el medioambiente.

Dentro de este marco de contradicciones, la producción agrícola del municipio de Marinilla, sustentada en la revolución verde, es cuestionada y se intenta volver a la práctica tradicional anterior que mantenía su base orgánica y que es aplicada actualmente por unos pocos agricultores, porque en 
la mayoría falta la memoria cultural que las valida. La práctica agrícola más reciente es la producción mixta, híbrida, definida como una forma de cultivo en la que se combinan aspectos tradicionales con elementos de la agricultura moderna, para el consumo de la familia y cubrimiento parcial de la demanda de la región.

La producción agrícola tiene una connotación especial, por cuanto convoca aspectos tales como: las prácticas de producción tradicionales y modernas, la seguridad alimentaria, la explotación adecuada de los recursos naturales y en relación con significaciones culturales que definen la responsabilidad en el consumo de alimentos, considerados en la base de la salud humana y la conservación del ecosistema. De esta manera, cada vez se da mayor importancia a la producción que no perjudique la vida del ser humano, como una forma de preservar también la vida del planeta, lo cual deriva en la definición de la calidad del producto, que debe ser certificada.

El sistema de producción agrícola de base orgánica, se ha planteado para un contexto de crisis alimentaria en el marco de un paradigma de desarrollo que agota los recursos naturales. Es necesario reconocer que el bienestar nutricional de todas las personas es una condición previa necesaria para el desarrollo de las sociedades. Debe constituir un objetivo fundamental del progreso en el desarrollo humano, reconocer la importancia que tiene la seguridad alimentaria y el papel que juegan los agricultores campesinos, las poblaciones indígenas y las comunidades que contribuyen con la producción de alimentos de manera orgánica. De igual manera, se convierte en un círculo virtuoso la producción alimentaria para disminuir los problemas de pobreza en el campo y mejorar los niveles nutricionales de los más pobres en el mundo para continuar abasteciendo la base alimentaria de la población mundial.

El tema de producción agrícola e industrial para la sostenibilidad se enmarca dentro de las preocupaciones de desarrollo sustentable y sostenible, a partir del círculo de Roma y del Informe Brundtland (ONU, 1987), sin embargo, la preocupación por las repercusiones en la salud humana, la vida del planeta y la seguridad alimentaria es relativamente reciente. Los temas relacionados con la garantía de la calidad en la producción agrícola toca estos aspectos que requieren mayor reflexión y profundidad, y compromiso de agentes gubernamentales y no gubernamentales para comprender y apoyar de manera integral estos temas.

Es necesario reforzar la estabilidad social y las políticas agrarias y económicas que protejan al pequeño agricultor que está en la base de la seguridad alimentaria y fomentar la sostenibilidad de la productividad agrícola. Por lo tanto, los proyectos de producción orgánica y de disminución de la pobreza en el campo, que desarrollan muchas asociaciones y organizaciones no gubernamentales, requieren de mayor acompañamiento por parte de instancias gubernamentales y del sector privado.

\section{BIBLIOGRAFÍA}

Alzate, Jorge, Vélez, Alejandro y Ramírez, Gustavo (1999). Programa de Creación, Declaratoria, Manejo y Administración de un distrito Agrario en el municipio de Marinilla-Antioquia. [En línea] Municipio de Marinilla, Universidad Nacional de Colombia, Municipios asociados del oriente antioqueño, Corporación Autónoma Regional Rionegro-Nare Cornare, 4lp. Disponible en: http://www.corpoceam.org/Distrito _ Agrario.pdf. [Consultado el 13 de septiembre de 2009|.

Blount M., Estefanía. Cerrar los ciclos de producción (1998). En: Abaco: Revista de Cultura y Ciencias Sociales, Madrid, n. ${ }^{\circ}$ 17-18, pp. 51-58.

Cumbre Mundial sobre alimentación (1996). Declaración. Adoptada en el día de Parlamentarios con ocasión de la Cumbre Mundial sobre la Alimentación. Unión interparlamentaria Roma. [En línea] Disponible en: http://www.ipu.org/splz-e/food.htm [Consultado el 9 de agosto de 2009]. 
Clements, Richard B. (1997). Guía completa de las normas ISO 14.000. [En línea] Barcelona. Disponible en: http://infoagro.net/shared/docs/a6/ACF21A.pdf [Consultado el 9 agosto de 2009].

Espinoza, Carolina (2004). Caracterización de la producción ecológica en Colombia. [En línea] IICA. 33 p. Disponible en: http://zeus.iica.ac.cr:8090/dspace/handle/123456789/99. [Consultado el 20 de septiembre de 2009].

FAO- Organización de la Naciones Unidas para la Agricultura y la Alimentación- (1996). Declaración de Roma sobre la Seguridad Alimentaria Mundial y Plan de la cumbre sobre la alimentación de Acción. Roma. [En línea] Disponible en: http://translate.google.com/translate ?hl=es\&langpair=en\%7Ces\&u=http://www.fao.org/ docrep/003/w3613e/w3613e00.htm. [Consultado el 13 de agosto de 2009].

FAO- Organización de la Naciones Unidas para la Agricultura y la Alimentación- (2001). Los mercados mundiales de frutas y verduras orgánicas, Roma. [En línea] Disponible en: http://www.fao.org/docrep/004/ yl669s/yl669s00.htm\#Contents. [Consultado el 8 de septiembre de 2009].

FAO- Organización de la Naciones Unidas para la Agricultura y la Alimentación - (2003). Código de prácticas de higiene para las frutas y hortalizas frescas. Codex Alimentarius. CAC/RCP 53-2003. [En línea]. Disponible en: http://www.codexalimentarius.net/ web/more _info.jsp?id_sta $=10200$ [Consultado el 15 de septiembre de 2009].

FAO, OMS- Organización de las Naciones Unidas para la Agricultura y la alimentación, y organización Mundial de la Salud, - (2004). Garantía de la inocuidad y calidad de los alimentos: Directrices para el fortalecimiento de los sistemas nacionales de control de los alimentos. [En línea] (s.f.) 94p Disponible en: http://www.who.int/ foodsafety/publications/capacity/en/Spanish_Guidelines_Food_control.pdf. [Consultado el 23 de agosto de 2009].

IFOAM- Federación Internacional De Movimientos De Agricultura Orgánica: Informe anual 2000. [En línea] Disponible en: http://translate.google.com/trans late?hl=es\&langpair $=$ en\%7Ces\&u $=$ http://www. ifoam.org/about_ifoam/inside_ifoam/pdfs/
IFOAM_Annual_Report_2000.pdf IConsultado el 15 de septiembre de 2009].

Forero, J. A (2003). Economía campesina y sistema alimentario en Colombia: Aportes para la discusión sobre seguridad alimentaria. En: Memorias Seminario regional Desarrollo rural y soberanía alimentaria. Universidad Nacional sede Medellín, Vamos Mujer, Ecofondo, CIER Y CORPOICA. Medellín: 20 y 21 Nov., Universidad Nacional.

Galeano, C., Alejandro (2001). De la Seguridad alimentaria a la soberanía alimentaria. En: Memorias, Colombia en el Fórum Social Mundial. Bogotá.

Jaramillo, Oscar (2008). Secretaría de Agricultura de Marinilla. Entrevista inédita. Marinilla, Antioquia

Mejía, M. G. (2003). Soberanía y seguridad alimentarias por la vía campesina. Retorno al campo. Medellín: En: Memorias Seminario regional Desarrollo rural y soberanía alimentaria. Universidad Nacional sede Medellín, Vamos Mujer, Ecofondo, CIER Y CORPOICA. Medellín: 20 y 21 Nov., Universidad Nacional.

Ministerio de Agricultura y Desarrollo rural (2002). Resolución número 00074 de 2002. Por la cual se establece el reglamento para la producción primaria, procesamiento, empacado, etiquetado, almacenamiento, certificación, importación y comercialización de productos agropecuarios ecológicos. Bogotá: 4 de abril, 24p. [En línea] Disponible en: http:// www.redlactea.org/resoluciones/Resolucion\%20 00074\%20de\%202002.pdf. [Consultado el 18 de agosto de 2009].

ONU-Organización de Naciones Unidas-(1987a). Informe de la Comisión Mundial sobre Medio Ambiente y Desarrollo. Asamblea 96 sesión plenaria, Código 42/187. Departamento de las Naciones Unidas de Asuntos económicos y Sociales (DESA). [En línea] Disponible en: http://translate.google.com/translate ?hl=es\&langpair=en\%7Ces\&u=http://www.un.org/ documents/ga/res/42/ares42-187.htm. [Consultado el 13 de agosto de 2009].

ONU-Organización de Naciones Unidas-(1987b). Our Common Future: Brundtland Report. En: Development and international economic cooperation: environment. Report of world commission on environment and development, 42a session, 374p. 
ONU- Organización de Naciones Unidas- (1992). Declaración de Rio, sobre el Medio Ambiente y Desarrollo. La conferencia de Naciones Unidas sobre el Medio Ambiente y Desarrollo. Rio de Janeiro. [En línea] Disponible en: http://translate.googleusercontent.com/tran slate_c?hl=es\&langpair =en\%7Ces\&rurl=trans late.google.com\&twu=1\&u=http://earthsummit. ca/documents/Rio _ Declaration_on _Environment_and_Development_1992.pdfEusg=ALkJ rhilczw0Zo8W29UcOCqlylTn4Y2zcA. IConsultado el 13 de agosto de 2009].

Polanco, D. N.; Polanco, R.; Velásquez, R. A (2005): Sistemas de Producción Sostenible, Gestión Ambiental participativa como aporte a la Construcción de Paz y Desarrollo Humano Sostenible en las Regiones de Tolima y Huila. Ibagué: Ecofondo.

Pons, Jean y Sivardiére (2002). Manual de Capacitación Certificación de Calidad de los Alimentos Orientada a Sellos de Atributos de Valor en Países de América Latina. Organización de las Naciones Unidas para la Agricultura y la Alimentación. Oficina regional para América latina y el Caribe. [En línea] Disponible en: http://www.fao.org/docrep/004/ad094s/ad094s00. htm\#Contents. [Consultado el 20 de octubre de 2009].

Prager M., Martín; Restrepo M., José M.; Ángel S., Diego Iván; Malagón M., Ricardo; Zamorano M., Adriana (2002). Agroecología: Una disciplina para el estudio y desarrollo de sistemas sostenibles de producción agropecuaria. Ed. Feriva, Bogotá, 250 p.

Robledo, J. E. (2003). Globalización y Seguridad alimentaria, En: Machado C. Absalón. (Comp.). Desarrollo Rural y Seguridad Alimentaria un reto para Colombia. Medellín: Universidad Nacional de Colombia, Sede.
Red de Desarrollo Rural y Seguridad Alimentaria. pp. 31-45.

Rodriguez M., Hernán D., Propuesta para la Producción Limpia en los Sistemas Agrícolas del Distrito Agrario de Marinilla (2000). Tesis Especialización en Gestión Agroambiental. Medellín: Universidad Nacional de Colombia, Noviembre. T0883

Rosas R., Antonio. (2003). Agricultura Orgánica Práctica. Alternativas Tecnológicas para la agricultura del futuro. Bogotá: Ediciones Arístides Gómez S. 285p.

Saborío, Guillermo. Y Delgado, Geovanny. (2006). La certificación en la agricultura orgánica (Respuestas a las preguntas más comunes): Costa Rica: Eco-Lógica. 19p. [En línea] Disponible en: http:// www.cedeco.or.cr/documentos/Certificacion.pdf. [Consultado el 21 de enero de 2010].

Sánchez, S. E (2005). La Agricultura Urbana, una estrategia de seguridad alimentaria para Colombia. En: Eolo: Revista Ambiental, Medellín, Vol. 05, n. 10, Oct., pp. 62-65.

Suaza S, Doris E (2008). Consolidación del distrito Agrario una experiencia piloto en el País, Municipio de Marinilla, Marinilla. [En línea] Disponible en: http://www. fontagro.org/projects/98_08_extension/documentos\%20proyecti\%20extensi\%c3\%b3n/08_ 02 anexomarinilladistritoagrario2.pdf. [Consultado el 15 de octubre de 2009].

Thomson, A y Metz, M. (1999). Implicaciones de las políticas económicas en la seguridad alimentaria. Manual de capacitación. Preparado para el Servicio de Apoyo para Políticas Agrarias. Dirección de Asistencia para las Políticas de la FAO y la Sociedad alemana de cooperación técnica (GTZ). Roma: FAO. 365 p. 
